\title{
B cell maturation antigen-specific CAR T cells are clinically active in multiple myeloma
}

\author{
Adam D. Cohen, ${ }^{1}$ Alfred L. Garfall, ${ }^{1}$ Edward A. Stadtmauer, ${ }^{1}$ J. Joseph Melenhorst, ${ }^{2}$ Simon F. Lacey, ${ }^{2}$ Eric Lancaster, ${ }^{3}$ Dan T. Vogl, \\ Brendan M. Weiss, ${ }^{1}$ Karen Dengel, ${ }^{2}$ Annemarie Nelson, ${ }^{2}$ Gabriela Plesa, ${ }^{2}$ Fang Chen, ${ }^{2}$ Megan M. Davis, ${ }^{2}$ Wei-Ting Hwang, ${ }^{4}$ \\ Regina M. Young, ${ }^{2}$ Jennifer L. Brogdon, ${ }^{5}$ Randi Isaacs, ${ }^{5}$ Iulian Pruteanu-Malinici, ${ }^{5}$ Don L. Siegel, ${ }^{2,6}$ Bruce L. Levine, ${ }^{2,6}$ \\ Carl H. June, ${ }^{2,6}$ and Michael C. Milone ${ }^{6}$ \\ 'Abramson Cancer Center, ${ }^{2}$ Center for Cellular Immunotherapies, ${ }^{3}$ Department of Neurology, and ${ }^{4}$ Department of Biostatistics, Epidemiology and Informatics, University of Pennsylvania, Philadelphia, Pennsylvania, \\ USA. ${ }^{5}$ Novartis Institute for Biomedical Research, Cambridge, Massachusetts, USA. ${ }^{5}$ Department of Pathology and Laboratory Medicine, University of Pennsylvania, Philadelphia, Pennsylvania, USA.
}

\begin{abstract}
BACKGROUND. CAR T cells are a promising therapy for hematologic malignancies. B cell maturation antigen (BCMA) is a rational target in multiple myeloma (MM).
\end{abstract}

\begin{abstract}
METHODS. We conducted a phase I study of autologous T cells lentivirally transduced with a fully human, BCMA-specific CAR containing $\mathrm{CD} 3 \zeta$ and 4-1BB signaling domains (CART-BCMA), in subjects with relapsed/refractory MM. Twenty-five subjects were treated in 3 cohorts as follows: cohort $1,1 \times 10^{8}$ to $5 \times 10^{8}$ CART-BCMA cells alone; cohort 2, cyclophosphamide (Cy) $1.5 \mathrm{~g} /$ $\mathrm{m}^{2}$ plus $1 \times 10^{7}$ to $5 \times 10^{7}$ CART-BCMA cells; cohort 3 , Cy $1.5 \mathrm{~g} / \mathrm{m}^{2}$ plus $1 \times 10^{8}$ to $5 \times 10^{8}$ CART-BCMA cells. No prespecified BCMA expression level was required.

RESULTS. CART-BCMA cells were manufactured and expanded in all subjects. Toxicities included cytokine release syndrome and neurotoxicity, which were grade 3-4 in 8 (32\%) and 3 (12\%) subjects, respectively, and reversible. One subject died at day 24 from candidemia and progressive myeloma, following treatment for severe cytokine release syndrome and encephalopathy. Responses (based on treated subjects) were seen in 4 of $9(44 \%)$ in cohort 1,1 of $5(20 \%)$ in cohort 2, and 7 of $11(64 \%)$ in cohort 3 , including 5 partial, 5 very good partial, and 2 complete responses, 3 of which were ongoing at 11, 14, and 32 months. Decreased BCMA expression on residual MM cells was noted in responders; expression increased at progression in most. Responses and CART-BCMA expansion were associated with CD4/CD8 T cell ratio and frequency of CD45RO-CD27+CD8 ${ }^{+}$ T cells in the premanufacturing leukapheresis product.
\end{abstract}

CONCLUSION. CART-BCMA infusions with or without lymphodepleting chemotherapy are clinically active in heavily pretreated patients with MM.

TRIAL REGISTRATION. NCT02546167.

FUNDING. University of Pennsylvania-Novartis Alliance and NIH.

Related Commentary: p. 2175

Conflict of interest: ADC reports research funding from Novartis, research funding and personal fees from Bristol-Meyers Squibb, and personal fees from Celgene, Kite Pharma, Janssen, Seattle Genetics, Oncopeptides, Takeda, Array Biopharma, and GlaxoSmithKline. ALG reports research funding from Novartis and Amgen, personal fees from Kite Pharma, and research funding and personal fees from Tmunity. EAS reports research funding from AbbVie and personal fees from Celgene, Takeda, Janssen, and Amgen. SFL reports research funding and other from Novartis and research funding from Tmunity. EL reports research funding from Grifols Inc. and personal fees from Merck Inc. and Novartis Inc. DTV reports personal fees from Karyopharm, Amgen, Millennium/Takeda, and Celgene, and research funding from GlaxoSmithKline. BMW reports research funding from Novartis, personal fees from Novartis and Alnylam, and research funding from Janssen and Prothena. BMW became an employee of Janssen Research and Development in October 2017. JJM, GP, RMY, and MCM report research funding from Novartis. JLB, RI, and IPM are employees of Novartis. DLS reports other from Poseida Therapeutics. BLL reports research funding from Novartis, personal fees from Avectas, Brammer Bio, Incysus, CRC Oncology/Cure Genetics, Novartis, Terumo, and Draper Labs, and other from Tmunity Therapeutics. CH] reports research funding from Novartis, and he is a scientific founder of Tmunity Therapeutics, for which he has founders stock but no income. ADC, ALC, EAS, JJM, SFL, EL, GP, FC, MMD, BLL, CHJ, and MCM hold or have pending patents (15/757,123, 17/042,129, 16/050,112, 62/586,834, 62/593,043, 62/752,010, 62/588,836) related to intellectual property licensed by the University of Pennsylvania to Novartis.

Role of funding source: This study was funded by a sponsored research agreement between the University of Pennsylvania and Novartis to develop chimeric antigen receptor (CAR) T cells for therapeutic use. Novartis provided the B cell maturation antigen CAR construct but did not dictate study design, conduct, or analysis. Some funds for correlative analyses were provided by NIH grant 1P01CA214278.

Copyright: (5) 2019, American Society for Clinical Investigation.

Submitted: November 21, 2018; Accepted: March 12, 2019; Published: April 29, 2019

Reference information: / Clin Invest. 2019;129(6):2210-2221, https://doi.org/10.1172/JCI126397. 


\section{Introduction}

Despite advances in multiple myeloma (MM) therapy, the disease remains incurable in most patients, and novel approaches are needed. B cell maturation antigen (BCMA) is a cell surface receptor expressed primarily by plasma cells. Its ligands are BAFF and APRIL, and it functions to maintain long-lived plasma cell homeostasis (1). BCMA is expressed consistently on MM cell lines and primary patient samples, though intensity of expression is variable (2-5). BCMA is shed from the surface of plasma cells by gamma-secretase-mediated cleavage (6), leading to a soluble form (sBCMA) that is detectable in circulation. Higher concentrations of sBCMA in patients with myeloma are associated with poorer clinical outcomes (7). Ligation of BCMA promotes MM cell proliferation, survival, and drug resistance $(8,9)$, and antibody-based blockade of BCMA signaling has anti-MM activity in preclinical models $(3,9)$. Thus, BCMA is a rational target for antimyeloma therapy.

Chimeric antigen receptors (CARs) provide novel specificity to $\mathrm{T}$ cells, coupling antibody-based extracellular domains to $\mathrm{T}$ cell signaling domains and costimulatory molecules. We previously reported that CD19-specific CAR T cells given after salvage autologous stem cell transplantation could prolong remission duration in some patients with relapsed/refractory MM $(10,11)$. Carpenter et al. demonstrated preclinical anti-MM activity using BCMAspecific CAR T cells (4), and adoptive transfer of autologous T cells expressing this murine BCMA CAR, following lymphodepletion with fludarabine and cyclophosphamide conditioning, induced objective responses in 4 of 12 heavily pretreated patients with MM. Responses were associated with significant but expected toxicities including cytokine release syndrome (CRS) and neurotoxicity (12), similar to those described with CD19-targeted CAR T cells in patients with B cell malignancies $(13,14)$.

We developed what we believe is a novel BCMA-targeted CAR with a fully human $\mathrm{scFv}$ (single chain variable fragment), fused to the hinge and transmembrane domain of CD8 and the human $4-1 \mathrm{BB}$ and $\mathrm{CD} 3 \zeta$ intracellular signaling domains and packaged in a lentiviral vector, with demonstrated preclinical activity (15). We now report results from our phase I clinical trial evaluating autologous $\mathrm{T}$ cells expressing this fully human BCMA-specific CAR (CART-BCMA) in relapsed/refractory myeloma patients, both with and without lymphodepleting chemotherapy.

CART-BCMA manufacturing *

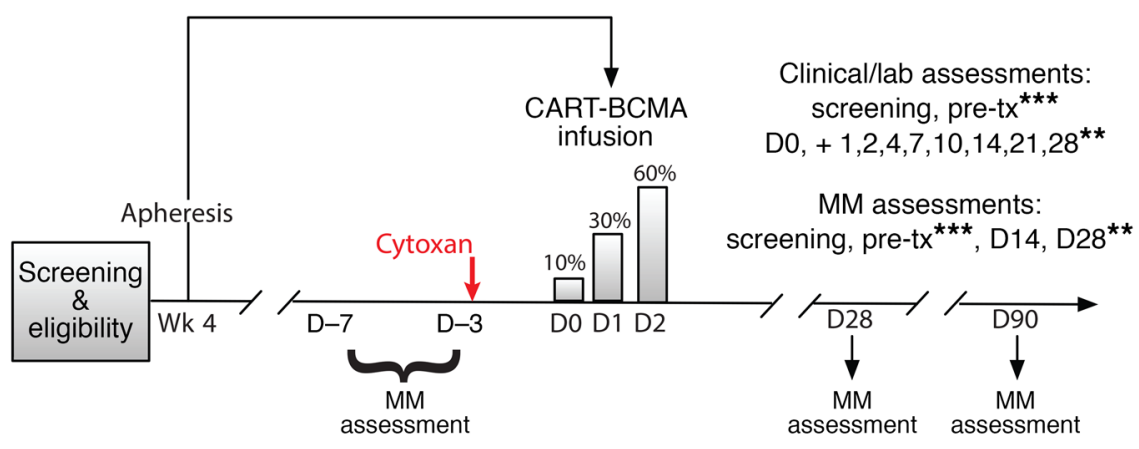

Figure 1. Treatment schema. Cytoxan indicates cyclophosphamide. *Patients may receive therapy during manufacturing to maintain disease control. ${ }^{* *}$ After first 28 days, follow-up is every 4 weeks up to 6 months, then every 3 months up to 2 years. ${ }^{* *}$ Pre-tx, pretreatment, 3 to 7 days before CART cell infusion.

\section{Results}

This clinical trial was a phase I, single-center, open-label study to evaluate the feasibility, safety, and clinical and biologic activity of manufacturing and administering CART-BCMA cells to patients with relapsed/refractory myeloma. We enrolled 3 sequential dose cohorts as follows: cohort $1,1 \times 10^{8}$ to $5 \times 10^{8}$ CART-BCMA cells alone; cohort 2, cyclophosphamide (Cy) $1.5 \mathrm{~g} / \mathrm{m}^{2}$ plus $1 \times 10^{7}$ to $5 \times$ $10^{7}$ CART-BCMA cells; cohort 3 , Cy $1.5 \mathrm{~g} / \mathrm{m}^{2}$ plus $1 \times 10^{8}$ to $5 \times 10^{8}$ CART-BCMA cells. Enrolled subjects underwent steady-state leukapheresis to collect T cells for CART-BCMA manufacturing, typically a 4-week process. Antimyeloma therapy could resume during manufacturing until 2 weeks prior to first CART-BCMA infusion. CART-BCMA cells were administered in an outpatient research unit over 3 days as split-dose intravenous infusions (10\% of dose given on day $0,30 \%$ on day 1 , and $60 \%$ on day 2 ). In cohorts 2 and 3, Cy was administered for lymphodepletion 3 days prior to first CART-BCMA infusion (Figure 1).

From November 2015 to December 2017, 34 subjects consented and 29 were eligible and commenced manufacturing, with 25 receiving CART-BCMA infusions. Four were not treated due to rapid disease progression and clinical deterioration during manufacturing and bridging therapy (Supplemental Figure 1; supplemental material available online with this article; https://doi. org/10.1172/JCI126397DS1). Baseline characteristics and prior lines of therapy are summarized in Table 1, with individual details shown in Supplemental Table 2. Subjects had a median of 7 prior lines of therapy, with $96 \%$ dual refractory to a proteasome inhibitor (PI) and immunomodulatory drug (IMID), 72\% refractory to daratumumab, and $44 \%$ penta-refractory to bortezomib, lenalidomide, carfilzomib, pomalidomide, and daratumumab. At least 1 high-risk cytogenetic abnormality was found in $96 \%$ of the subjects, and $68 \%$ of subjects had either deletion 17 p or a TP53 mutation. Baseline tumor burden was high (median $65 \%$ myeloma cells on bone marrow biopsy), and $28 \%$ had extramedullary disease.

For all subjects, the minimum target goal of CART-BCMA cells was successfully manufactured and formulated, though 1 subject required 2 leukaphereses and manufacturing attempts. Final products comprised a median of $97 \% \mathrm{CD}^{+} \mathrm{T}$ cells, with median CD4/CD8 ratio of 1.7. Twenty-one subjects received all 3 planned CART-BCMA infusions, with 4 receiving $40 \%$ of planned dose (third infusion held due to early CRS). Further details of manufacturing, product characteristics, and dosing for each subject are shown in Supplemental Table 3.

Grade 3 or higher adverse events, regardless of attribution, were seen in 24 of 25 subjects $(96 \%)$ and are summarized in Table 2, with individual adverse events for each subject listed in Supplemental Table 4. Twentyfour of 25 subjects (96\%) were admitted to the hospital a median of 4 days (range 1-28 days) after first CART-BCMA infusion, with longer time to admission in cohort 2 $\left(1 \times 10^{7}\right.$ to $5 \times 10^{7}$ CART-BCMA cells, median 8 days) than cohorts 1 and $3\left(1 \times 10^{8}\right.$ to $5 \times 10^{8}$ CART-BCMA cells, median 3 days for both). CRS was observed in 22 of 25 subjects (88\%), 


\section{Table 1. Subject characteristics}

\begin{tabular}{|c|c|}
\hline Characteristic $(n=25)$ & Median (range) or $\%$ \\
\hline Age, years & $58(44-75)$ \\
\hline Sex & $68 \%$ male; $32 \%$ female \\
\hline Median time from diagnosis, years & $4.6(1.8-14.5)$ \\
\hline High-risk cytogenetics ${ }^{A}$ & $96 \%$ \\
\hline Del 17p or TP53 mutation & $68 \%$ \\
\hline Prior lines of therapy, no. & $7(3-13)$ \\
\hline Len / Bort / Pom / Carf B / Dara, \% exposed & $100 \% / 100 \% / 92 \% / 96 \% / 76 \%$ \\
\hline Len / Bort / Pom / Carf / Dara, \% refractory & $76 \% / 88 \% / 88 \% / 80 \% / 72 \%$ \\
\hline Dual- / Quad- / Penta-refractory & $96 \% / 56 \% / 44 \%$ \\
\hline Prior autologous SCT & $92 \%$ \\
\hline Extramedullary disease & $28 \%$ \\
\hline Bone marrow plasma cells & $65 \%(0-95)$ \\
\hline $\begin{array}{l}\text { Absolute } \mathrm{CD}^{+} \mathrm{T} \text { cell count preleukapheresis, }{ }^{\complement} \\
\text { cells/ } \mu \mathrm{l}\end{array}$ & $538(151-1529)$ \\
\hline Day 0 LDH, U/I & $175(75-385)$ \\
\hline Day 0 serum creatinine, $\mathrm{mg} / \mathrm{dl}$ & $0.93(0.55-2.87)$ \\
\hline Day 0 hemoglobin, g/dl & $9.1(6.4-12.4)$ \\
\hline Day 0 platelets, $\times 10^{3} / \mu \mathrm{l}$ & $128(13-316)$ \\
\hline \multicolumn{2}{|c|}{$\begin{array}{l}\text { AIncludes complex karyotype, gain 1q, deletion 17p, t(14;16), and/or t(4;14). } \\
{ }^{B} \text { Includes } 1 \text { patient who received oprozomib. }{ }^{\circ} n=23 \text { (subjects } 01 \text { and } 02 \\
\text { did not have prepheresis T cell counts done). Normal range, 900-3245 } \\
\text { cells/ } \mu \text { I. Bort, bortezomib; Carf, carfilzomib; Dara, daratumumab; Del, } \\
\text { deletion; Dual-refractory, refractory to both a proteasome inhibitor (PI) } \\
\text { and immunomodulatory agent (IMID); LDH, lactate dehydrogenase; Len, } \\
\text { lenalidomide; Penta-refractory, refractory to } 2 \text { PIs, } 2 \text { IMIDs, and Dara; Pom, } \\
\text { pomalidomide; Quad-refractory, refractory to } 2 \text { Pls and } 2 \text { IMIDs; SCT, stem } \\
\text { cell transplant. }\end{array}$} \\
\hline
\end{tabular}

and was grade $3-4$ on the Penn grading scale (16) (Supplemental Table 1) in 8 (32\%) subjects, all of whom were treated at the $1 \times$ $10^{8}$ to $5 \times 10^{8}$ dose. Median time to CRS onset was 4 days (range 1-11 days), with a median duration of 6 days (range 1-18 days), and median duration of hospitalization of 7 days (range 0-40 days). CRS was associated with elevations in ferritin and C-reactive protein, as previously described (14). Seven subjects (28\%) received IL-6 blockade with either tocilizumab $(n=6)$ or siltuximab $(n=1)$.

Neurotoxicity was seen in 8 of 25 subjects (32\%), and was mild (grade 1-2) in 5 (transient confusion and/or aphasia) subjects. Three (12\%) had grade 3-4 encephalopathy including 1 subject (subject 03) in cohort 1 with a dose-limiting toxicity (DLT) of PRES (posterior reversible encephalopathy syndrome) with severe obtundation, recurrent seizures, and mild cerebral edema on MRI that fully resolved after treatment with high-dose methylprednisolone (1 g/day times 3) and cyclophosphamide $1.5 \mathrm{~g} / \mathrm{m}^{2}$. The others had no objective changes on MRI. All 3 subjects with severe neurotoxicity had high tumor burden ( 2 with extramedullary disease), had received a dose of $5 \times 10^{8}$ CART-BCMA cells, and had grade 3 or 4 CRS. A summary of CRS and neurotoxicity based on cohort is provided in Supplemental Table 5 . The other DLT was grade 3 cardiomyopathy and grade 4 spontaneous hemothorax in subject 27 (cohort 3) in the setting of CRS, coagulopathy, thrombocytopenia, and extensive myelomatous rib lesions. Of note, this subject had received siltuximab (anti-IL-6 $\mathrm{mAb}$ ) and low-dose steroids for grade $3 \mathrm{CRS}$ due to concurrent grade
3 encephalopathy and treating physician's concern that tocilizumab, which blocks the IL- 6 receptor and can cause a spike in serum IL-6 concentration (17), might exacerbate the subject's neurotoxicity. All of these toxicities fully resolved. No unexpected off-target toxicities were observed.

There was one grade 5 event during the study. Subject 08 (cohort 1) was a 71-year-old man with IgA lambda MM with complex karyotype. By the time of CART-BCMA infusion, he was progressing rapidly through bridging therapy with worsening kidney function, pancytopenia, and extensive extramedullary disease. He tolerated all 3 CART-BCMA infusions and was admitted on day 4 with grade $1 \mathrm{CRS}$, grade 1 delirium, and possible pneumonia. $\mathrm{He}$ was stable initially with antibiotics and supportive care, but on day 14 , he developed grade 4 CRS with hypoxemia, distributive shock and renal failure requiring dialysis, as well as grade 4 encephalopathy requiring intubation. Tocilizumab and steroids were given with improvement, and by day 19 , he was extubated with discontinuation of dialysis and steroids. He then worsened on days 21-22 with recurrent pressor-dependent hypotension, hypoxemia, worsening renal function, and confusion. He was reintubated, and his condition worsened despite additional tocilizumab and steroids. Blood cultures identified candidemia. Restaging labs revealed a serum M-spike of $5.1 \mathrm{~g} / \mathrm{dl}$ (increased from $2.5 \mathrm{~g} / \mathrm{dl}$ pretreatment), and serum-free lambda light chains $2544 \mathrm{mg} / \mathrm{l}$ (increased from $882 \mathrm{mg} / \mathrm{l}$ pretreatment), along with $13 \%$ circulating plasma cells. His family opted to withdraw aggressive support and pursue comfort care only, and the subject died at day 24 . No other deaths occurred during the study.

Objective responses (partial response [PR] or better) were confirmed in 4 of 9 subjects (44\%) in cohort 1, 1 of 5 subjects (20\%) in cohort 2, and 7 of 11 subjects $(64 \%)$ in cohort 3 (Figure $2 \mathrm{~A}$ ), including 5 PR, 5 very good partial response (VGPR), 1 complete response (CR), and 1 stringent complete response (sCR). The overall response rate was 12 of 25 (48\%), with 11 of 20 subjects $(55 \%)$ responding at the more effective dose of $1 \times 10^{8}$ to 5 $\times 10^{8}$ CART-BCMA cells. Five additional subjects had minimal response (MR). The overall response rate by intention-to-treat, based on the 29 subjects who were eligible and underwent apheresis, was 12 of 29 (41\%). Four of 7 subjects with extramedullary disease responded (Figure 2B). Four subjects (subjects 01, 03, 15, 19) had no detectable myeloma by flow cytometry (estimated sensitivity $10^{-5}$ ) from postinfusion marrow aspirates at month 1 and/or 3. Median time to first response was 14 days. Based on Kaplan-Meier estimates, median duration of response was 124.5 days (range 29 -939+) (Supplemental Figure 2). At time of data cut-off, 3 subjects (subjects 01, 19, 33) remained progression free at 953, 427, and 322 days (roughly 32, 14, and 11 months), respectively. All other subjects have progressed, and median progression-free survival (PFS) is 65, 57, and 125 days for cohorts 1,2 , and 3, respectively (Supplemental Figure 2). At time of data cut-off, 13 subjects had expired, with median overall survival of 502 days for all subjects (Supplemental Figure 2), and 359 days, 502 days, and not reached for cohorts 1, 2, and 3, respectively (Figure 2C).

All infused subjects had detectable CART-BCMA cells in peripheral blood by qPCR (Figure 3, A-C), and 24 of 25 had detectable $\mathrm{CAR}^{+} \mathrm{T}$ cells by flow cytometry (Supplemental Figures 4-6; Supplemental Figure 3 for representative staining). Expan- 
Table 2. Grade 3 or higher adverse events, regardless of attribution

\begin{tabular}{|c|c|c|c|c|}
\hline Event & $\begin{array}{c}\text { Grade } 3 \\
n\end{array}$ & $\begin{array}{c}\text { Grade } 4 \\
n\end{array}$ & $\begin{array}{c}\text { Grade } 5 \\
n\end{array}$ & $\begin{array}{c}\text { All } \\
n(\%)\end{array}$ \\
\hline Leukopenia & 3 & 8 & 0 & $11(44)$ \\
\hline Neutropenia & 2 & 9 & 0 & $11(44)$ \\
\hline Lymphopenia & 3 & 6 & 0 & $9(36)$ \\
\hline Cytokine release syndrome & 7 & 1 & 0 & $8(32)$ \\
\hline Thrombocytopenia & 3 & 4 & 0 & $7(28)$ \\
\hline Hypophosphatemia & 7 & 0 & 0 & $7(28)$ \\
\hline Febrile neutropenia & 6 & 0 & 0 & $6(24)$ \\
\hline Hyponatremia & 6 & 0 & 0 & $6(24)$ \\
\hline Anemia & 4 & 1 & 0 & $5(20)$ \\
\hline Hypocalcemia & 3 & 2 & 0 & $5(20)$ \\
\hline AST increased & 2 & 1 & 0 & $3(12)$ \\
\hline Hypokalemia & 2 & 1 & 0 & $3(12)$ \\
\hline Neurotoxicity ${ }^{A}$ & 1 & 2 & 0 & $3(12)$ \\
\hline Hypertension & 3 & 0 & 0 & $3(12)$ \\
\hline Lung infection & 2 & 0 & 0 & $2(8)$ \\
\hline Hypoalbuminemia & 2 & 0 & 0 & $2(8)$ \\
\hline Supraventricular tachycardia & 1 & 0 & 0 & $1(4)$ \\
\hline Papilledema & 1 & 0 & 0 & $1(4)$ \\
\hline Abdominal pain & 1 & 0 & 0 & $1(4)$ \\
\hline Death NOSB & 0 & 0 & 1 & $1(4)$ \\
\hline Fatigue & 1 & 0 & 0 & $1(4)$ \\
\hline Infections - other (fungemia) & 0 & 1 & 0 & $1(4)$ \\
\hline Sepsis & 0 & 1 & 0 & $1(4)$ \\
\hline Urinary tract infection & 1 & 0 & 0 & $1(4)$ \\
\hline Alk phos increased & 1 & 0 & 0 & $1(4)$ \\
\hline Ejection fraction decreased & 1 & 0 & 0 & $1(4)$ \\
\hline Fibrinogen decreased & 1 & 0 & 0 & $1(4)$ \\
\hline Hyperglycemia & 0 & 1 & 0 & $1(4)$ \\
\hline Hypoglycemia & 1 & 0 & 0 & $1(4)$ \\
\hline Pleural effusion & 1 & 0 & 0 & $1(4)$ \\
\hline Pleural hemorrhage & 0 & 1 & 0 & $1(4)$ \\
\hline
\end{tabular}

Highest grade toxicity experienced by subject is reported in table.

${ }^{A}$ Neurotoxicity includes 1 subject with grade 3 seizures, grade 4 delirium, and grade 4 reversible posterior leukoencephalopathy syndrome (RPLS) (also known as posterior reversible encephalopathy syndrome [PRES]); 1 subject with grade 3 delirium and grade 4 encephalopathy; and 1 subject with grade 3 encephalopathy. ${ }^{B}$ Death NOS (not otherwise specified) in subject 08 with candidemia and rapidly progressive myeloma, whose family chose to pursue comfort measures only. $n$, number of subjects who had the event; Alk phos, alkaline phosphatase; AST, aspartate aminotransferase.

sion generally peaked at day 10-14, and appeared most uniform in cohort 3 with Cy conditioning and the higher dose of CARTBCMA cells, while it was more heterogeneous in cohorts 1 and 2, though this difference did not meet statistical significance (Figure 3D). Despite a predominance of $\mathrm{CD} 4^{+} \mathrm{T}$ cells in the infused product, CART-BCMA cells circulating in blood were predominantly $\mathrm{CD} 8^{+}$, and were highly activated, with a median of $94 \%$ (range $21 \%-94 \%$ ) of $\mathrm{CAR}^{+} \mathrm{CD}^{+}$cells expressing HLA-DR during peak expansion (Supplemental Table 6). CART-BCMA levels in marrow aspirates generally mirrored those in peripheral blood, and also were elevated in pleural fluid and cerebrospinal fluid for subject 03 , and pleural fluid from subject 27, demonstrating widespread trafficking (Supplemental Table 7). Following peak expansion, CART-BCMA cell levels by qPCR declined in a log-linear fashion in the majority of patients (Figure $3, \mathrm{~A}-\mathrm{C}$ ), and were still detectable 3 months after infusion in 20 of $20(100 \%)$ subjects tested, and at 6 months in 14 of 17 (82\%) tested. Subject 01 (in stringent CR) continued to have detectable cells by qPCR when last tested 2.5 years after infusion.

We quantified 30 cytokines in peripheral blood serum before and after CART-BCMA infusion. Nineteen of these were increased more than 5 -fold over baseline in more than 1 subject, with the most frequent increases observed for IL-6, IL-10, monokine induced by interferon-gamma (MIG, CXCL9), IP-10, IL-8, GM-CSF, and IL-1 receptor antagonist (IL-1RA) (Supplemental Figure 7). More severe CRS (grade 3-4, or grade 2 receiving tocilizumab) was associated with increases in multiple cytokines (Supplemental Table 8), most significantly with IFN- $\gamma$, IL-2 receptor alpha (IL2-R $\alpha$ ), macrophage inflammatory protein 1 alpha (MIP1a), and IL-15 (Figure 4, A-D). Neurotoxicity was most strongly associated with increases in IFN- $\gamma$, IL-1RA, and MIP-1 $\alpha$ (Figure 4, E-G, see Supplemental Table 9 for full analysis). Peak foldincrease in IL-6 was strongly associated with both severe CRS and neurotoxicity (Supplemental Table 8, Supplemental Table 9); however, tocilizumab and siltuximab administration can artificially elevate serum IL-6 levels (17). When only IL-6 values obtained prior to administration of tocilizumab $(n=6)$ or siltuximab $(n=1)$ were included in the analyses, the association of peak IL-6 fold-increase with severe CRS and neurotoxicity was still present, but less statistically robust (Figure 4, $\mathrm{H}$ and I). We did not observe significant differences in peak fold cytokine increases between cohorts 1 and 3, which received the same dose of CART-BCMA cells with or without Cy conditioning, respectively (Supplemental Figure 7).

We also assessed serum concentration of SBCMA, as well as its ligands BAFF and APRIL. Compared with a panel of healthy donors (HDs), enrolled subjects had significantly elevated sBCMA and reduced APRIL levels at baseline, with high variability among subjects (Figure 5A). BAFF concentrations in subjects were not significantly different from HDs. Serial assessments of serum sBCMA showed decreases following CART-BCMA infusions, which were more pronounced in responding compared with nonresponding subjects (Figure 5B). sBCMA increased overall as both responders and nonresponders developed progressive disease, though the 3 long-term responders maintained low sBCMA concentrations (Supplemental Figure 8), confirming serum sBCMA concentration as a useful adjunctive biomarker for assessing myeloma disease response and progression (7).

Twenty subjects were evaluable for BCMA surface expression on MM cells by flow cytometry performed on fresh marrow aspirates prior to treatment, and all had detectable BCMA expression, though intensity varied (median MFI, 3741; range 206-24,842; see Supplemental Figure 9 for representative gating). Of 18 subjects with evaluable serial BCMA expression (Figure $5 \mathrm{C}$ ), either at 1 month $(n=16), 3$ months $(n=8)$, and/or 5.5 months $(n=1), 12$ $(67 \%)$ had a decline in BCMA intensity at least at 1 postinfusion time-point, including 8 of 9 responders and 4 of 9 nonrespond- 
$\mathbf{A}$

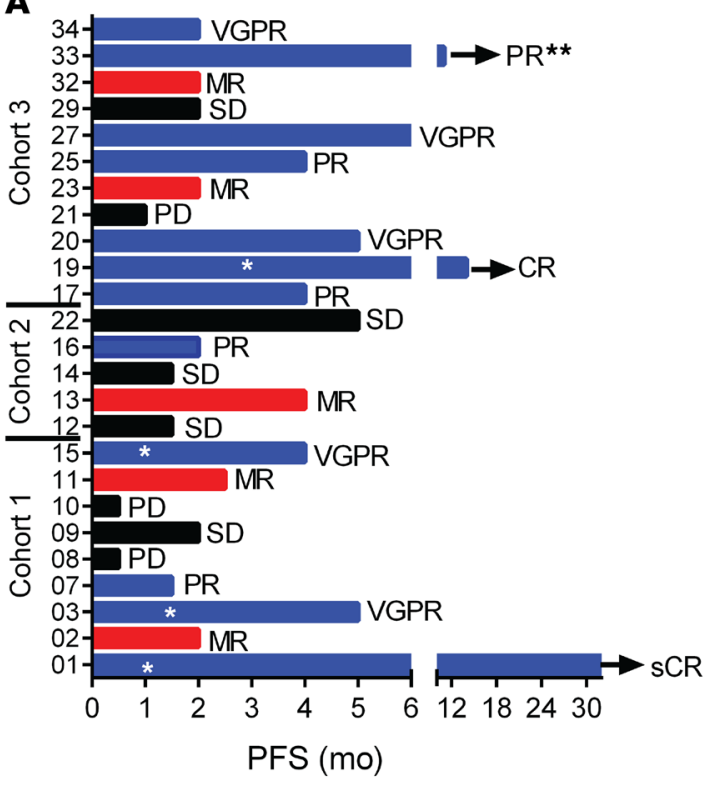

B

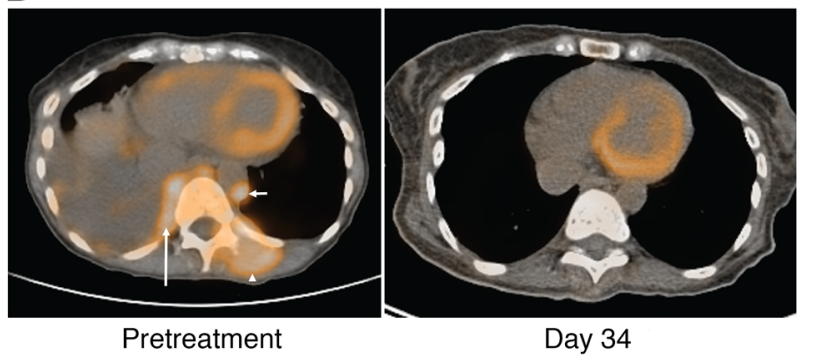

C

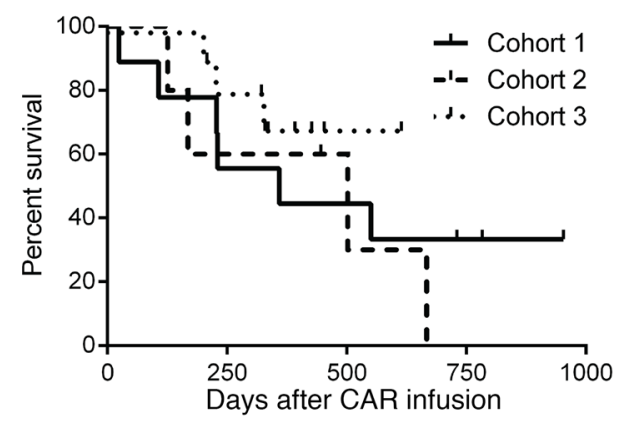

Figure 2. Clinical outcomes. (A) Swimmer's plot showing best response and progression-free survival (PFS) for each subject in cohort 1 ( $1 \times 10^{8}$ to $5 \times 10^{8}$ CART-BCMA cells alone), cohort 2 (Cy plus $1 \times 10^{7}$ to $5 \times 10^{7}$ CART-BCMA cells), and cohort 3 (Cy plus $1 \times 10^{8}$ to $5 \times 10^{8}$ CART-BCMA cells). Arrow indicates ongoing response. Blue bars represent PR or better; red bars, MR; black bars, no response (SD or PD). * Minimal residual disease (MRD), negative by flow cytometry (estimated sensitivity 1 in $10^{-5}$ cells). ${ }^{*}$ Has negative serum and urine immunofixation and negative bone marrow biopsy but residual retroperitoneal lymph nodes (LN), known to contain myeloma by prior biopsy, that decreased in size by more than 50\% and became FDG-negative on PET/CT but did not disappear. Repeat LN biopsy not performed. (B) PET/CT scan images for subject 03 showing resolution of extramedullary disease (arrows) and malignant pleural effusion after treatment. (C) Overall survival (OS) based on cohort, Kaplan-Meier plot. CR, complete response; MR, minimal response; PR, partial response; VGPR, very good partial response; PD, progressive disease; sCR, stringent complete response; SD, stable disease.

ers (Figure 5D). BCMA intensity was lowest on residual MM cells 1 month after CART-BCMA, and increased back toward baseline in most, but not all, subjects with subsequent testing. Individual subject details are provided in Supplemental Table 10. Neither progression-free nor overall survival was significantly associated with baseline BCMA MFI (Supplemental Figure 10).

Responses were significantly associated with peak expansion by qPCR (median 75,339 copies/ $\mu$ g DNA for $\geq$ PR vs. 6368 copies/ $\mu \mathrm{g}$ for $\angle \mathrm{PR}, P=0.0002$ ), as well as with persistence over the first 28 days, as measured by the area under the curve $\left(\mathrm{AUC}_{0-28 \mathrm{~d}}\right.$ ) (median 561,796 copies $\times$ days/ $\mu$ g DNA for $\geq P R$ vs. 52,391 copies $\times$ days $/ \mu g$ DNA for $\angle \mathrm{PR}, P=0.0002$ ) (Figure 6 , A and B). Both expansion and response were more likely in the setting of more severe CRS (grade 3-4 or grade 2 requiring tocilizumab) (Figure 6, C and D). Neither expansion nor response was significantly associated with age, years from diagnosis, number of prior lines of therapy, presence of del17p or TP53 mutation, being penta-refractory, most recent therapy prepheresis, bone marrow MM cell percentage, baseline serum sBCMA concentration, or MM cell BCMA intensity (Supplemental Figure 11, Supplemental Figure 12).

In order to explore other pretreatment characteristics potentially associated with expansion and/or response, we analyzed features of the CART-BCMA product before, during, and at the end of manufacturing. We found that a higher CD4/CD8 T cell ratio in the leukapheresis product, premanufacturing, was associated with greater in vivo CART-BCMA expansion (Figure 6E), and to a lesser degree, response (Figure 6F), whereas absolute $\mathrm{CD}^{+}, \mathrm{CD}^{+}$, or $\mathrm{CD}^{+}{ }^{+} \mathrm{T}$ cell numbers in the leukapheresis product, or $\mathrm{CD} 4 / \mathrm{CD} 8$ ratio in the final CART-BCMA product at the end of manufacturing was not (data not shown). Fold expansion of seeded cells during manufacturing also correlated with in vivo CART-BCMA expansion (Figure 6G), suggesting that in vitro proliferative capacity may predict for in vivo activity. Finally, our previous analyses in CLL patients receiving CD19-directed CAR $\mathrm{T}$ cells demonstrated that deeper clinical responses were associated with a higher percentage of $\mathrm{CD} 27^{+} \mathrm{CD} 45 \mathrm{RO}^{-} \mathrm{CD} 8^{+} \mathrm{T}$ cells within the leukapheresis product (18), a phenotype that identifies primarily naive but also includes stem cell memory $\mathrm{T}$ cell populations (19). We examined $\mathrm{CD} 8^{+} \mathrm{T}$ cells within the leukapheresis products in subjects treated with CART-BCMA cells and similarly found that subjects with higher frequencies of $\mathrm{CD} 27^{+} \mathrm{C}-$ $\mathrm{D}^{2} 5 \mathrm{RO}^{-} \mathrm{CD} 8^{+} \mathrm{T}$ cells were more likely to have robust in vivo expansion and clinical response (Figure 6, H and I).

\section{Discussion}

CAR T cell therapy is emerging as a promising therapeutic option for B cell malignancies, with the potential for durable disease control following a single treatment, differentiating it from other therapies that require repeated and/or continuous administration. In this report, we demonstrate the potential of CAR T cell therapy in advanced refractory myeloma, with 12 of 25 subjects (48\%) achieving a partial response or better, including 7 of $11(64 \%)$ treated 
A

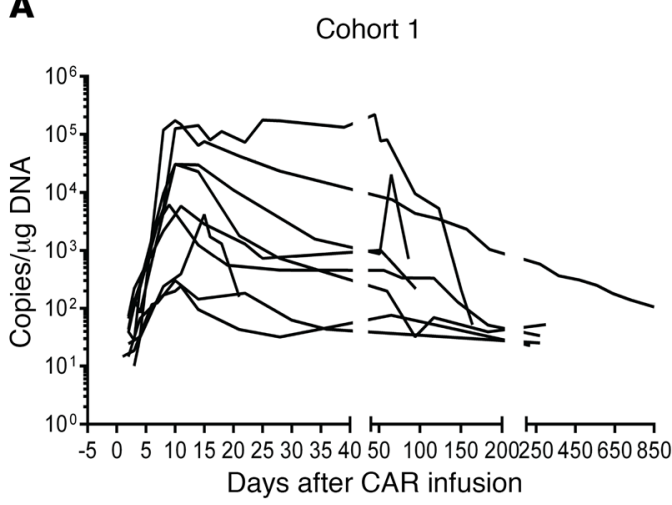

C

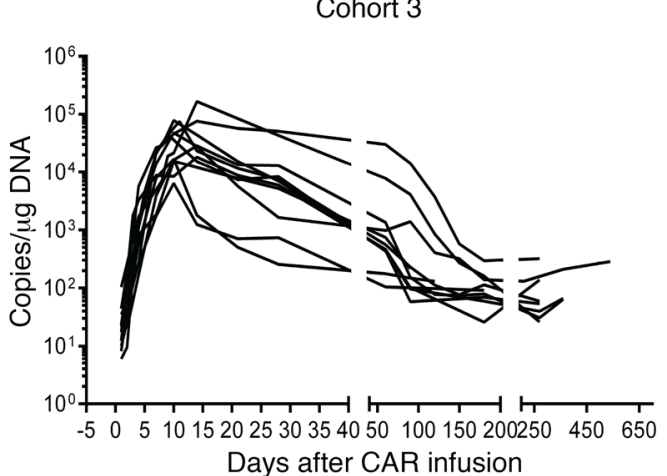

B

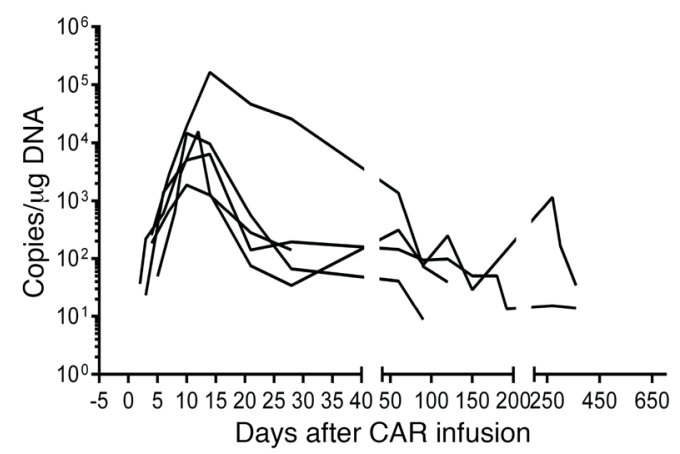

D

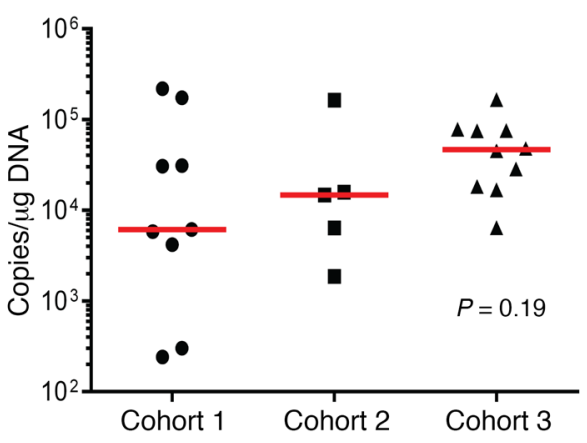

Figure 3. CART-BCMA expansion and persistence. (A-C) CART-BCMA cell levels over time in peripheral blood for each cohort are depicted, as measured by quantitative PCR for CAR sequence. (D) Peak CART-BCMA levels by qPCR for each subject are shown (except subject 34, for whom peak data were not available). Median peak CART-BCMA levels (red bars) were not significantly different between cohorts (Kruskal-Wallis test, $P=0.19$ ). in cohort 3 with lymphodepleting chemotherapy and the higher $\left(>10^{8}\right)$ dose of CART-BCMA cells. Three subjects had ongoing remissions longer than 11 months after CART-BCMA therapy, including one ongoing $\mathrm{sCR}$ at 2.5 years. This is notable given the highly adverse biological features of the enrolled subjects' myeloma, including high tumor burden, rapidly progressing disease, and high-risk genetics, and further validates BCMA as a highly attractive target in myeloma. Importantly, this activity was seen despite the fact that our study, unlike the previously reported NCI study, did not exclude patients with low BCMA expression or high tumor burden, and used either no lymphodepletion or Cy alone, compared with Cy plus fludarabine in the prior study $(12,20)$. We successfully manufactured CAR T cell products from all apheresed subjects, and saw engraftment in all subjects as well, though peak levels and persistence of CAR T cells were variable.

Myeloma has long been associated with quantitative and functional deficits in T cells, particularly in more advanced, refractory disease, with inverted CD4/CD8 $\mathrm{T}$ cell ratios, impaired ex vivo antitumor activity, and acquisition of an exhausted or senescent phenotype (21-23). Responses correlated with degree of in vivo expansion in our study, which in turn was associated with higher premanufacturing CD4/CD8 $\mathrm{T}$ cell ratio, premanufacturing frequency of $\mathrm{CD}^{2} 5 \mathrm{RO}^{-} \mathrm{CD} 27^{+} \mathrm{CD}^{+} \mathrm{T}$ cells, and magnitude of in vitro proliferation during manufacturing. This suggests that more effective CART-BCMA products may be derived from subjects with a less differentiated, more naive and/or stem cell memorylike $\mathrm{T}$ cell compartment, as previously observed in a CLL trial at our center using CD19-directed CAR T cells (18). Confirmation with larger numbers and other CAR $\mathrm{T}$ cell products is nec- essary, but these data suggest that pretreatment phenotypic and/or functional $\mathrm{T}$ cell characteristics may aid in the prediction of response to CART-BCMA therapy. They also suggest that treatment of patients earlier in the course of their disease, when T cells may be intrinsically "fitter," or modifying manufacturing techniques to generate more phenotypically favorable $\mathrm{CAR}^{+} \mathrm{T}$ cells, may be more effective. Alternatively, routine harvesting and storage of $\mathrm{T}$ cells early in disease course (e.g., in first remission) for later use in CAR manufacturing could be considered.

Successful adoptive transfer of tumor-specific $\mathrm{T}$ cells, including CAR T cells, in humans has most commonly followed some form of lymphodepleting conditioning (13, 14, 24-26), which has been demonstrated to enhance T cell-mediated antitumor immunity via multiple potential mechanisms, including reduction of cellular "sinks" leading to increased availability of homeostatic cytokines, and depletion of suppressor cell populations, among others (27). Our study demonstrates that lymphodepletion is not absolutely required for robust and sustained CAR T cell expansion and clinical activity, as seen with subjects 01 and 03 in cohort 1 . However, we observed short-term expansion more consistently in cohort 3 , where subjects received $\mathrm{Cy}$ conditioning, compared with cohort 1 (Figure 3), demonstrating an effect of lymphodepletion on CAR T cell kinetics following adoptive transfer. Given that studies showing incremental increases in the intensity of the conditioning lead to enhanced engraftment and clinical outcomes $(13,24)$, it is possible that modifying the lymphodepletion (e.g., adding fludarabine to $\mathrm{Cy}$ ) may further augment the activity of CART-BCMA cells, and we are testing this in ongoing trials. 
A

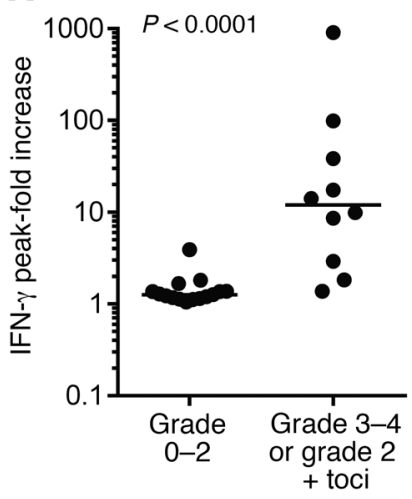

D

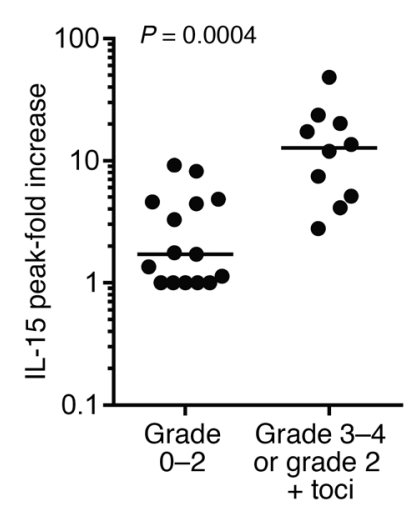

G

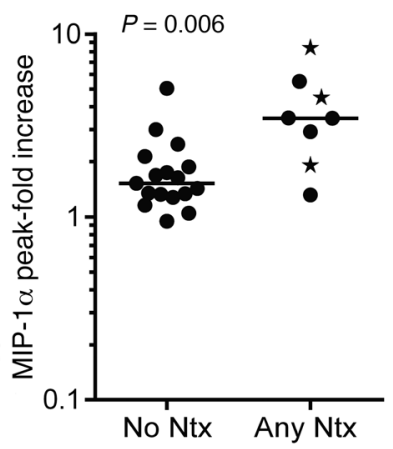

B

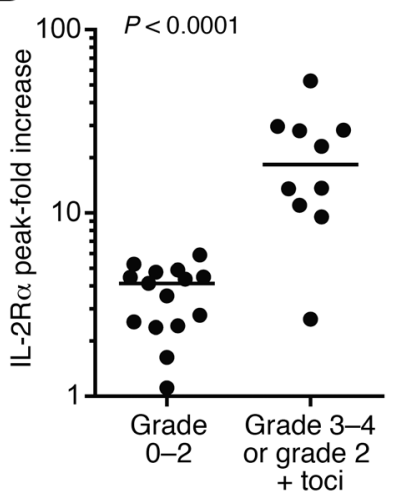

E

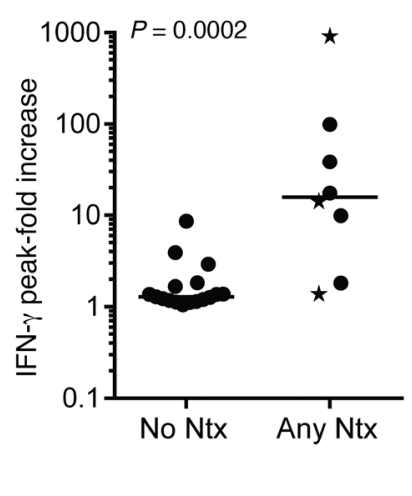

H

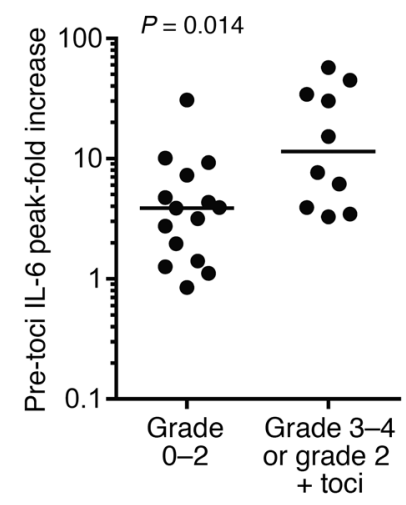

C

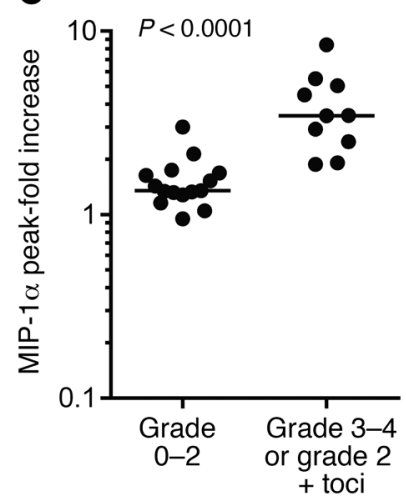

$\mathbf{F}$

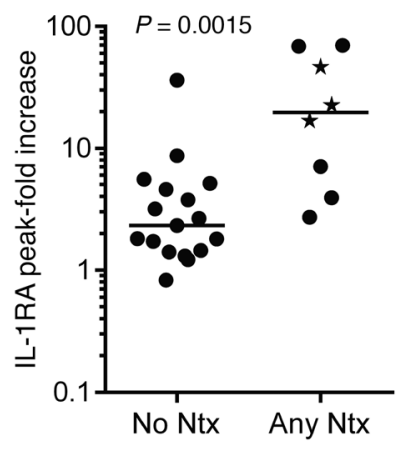

I

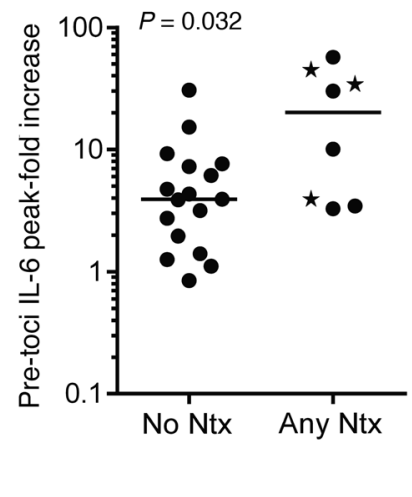

Figure 4. Serum cytokines associated with CRS severity and neurotoxicity. Serum cytokine concentrations (in pg/ $\mathrm{ml}$ ) through day 28 were measured by Luminex assay. (A-D) The median peak fold-increase over baseline for each cytokine was compared between subjects with no CRS, grade 1 CRS, or grade 2 CRS not receiving tocilizumab (CRS grade $0-2$ ) and those with grade $3-4$ CRS or grade 2 CRS receiving tocilizumab (CRS grade 3-4 or grade $2+$ toci). The cytokines most significantly associated with CRS severity were (A) IFN- $\gamma$, (B) IL-2R $\alpha$, (C) MIP-1 $\alpha$, and (D) IL-15. (E-C) Median peak fold-increase over baseline for each cytokine was compared between subjects with no neurotoxicity (No Ntx) and those with any grade of neurotoxicity (Any Ntx). The cytokines most significantly associated with neurotoxicity were (E) IFN- $\gamma$, (F) IL-1RA, and (C) MIP-1 $\alpha$. (H-I) Peak fold-increase in IL-6 was less significantly associated with severe CRS (H) or neurotoxicity (I) when only pretocilizumab/siltuximab values were included. Stars depict subjects with grade 3-4 neurotoxicity. Exact $P$ value by Mann-Whitney test is shown. Because the statistical analyses performed here are exploratory and hypothesis-generating in nature, no adjustment of the $P$ values was made for multiple comparisons. Horizontal lines depict medians. IFN- $\gamma$, interferon gamma; IL-1RA, interleukin 1 receptor antagonist; IL-2R $\alpha$, interleukin 2 receptor alpha; IL-6, interleukin 6; IL-15, interleukin 15; MIP-1 $\alpha$, macrophage inflammatory protein 1 alpha.
An important unanswered question for BCMA-targeted CAR T cells is whether there is a threshold of BCMA expression on MM cells required for optimal recognition and killing. In the previously reported NCI trial, 52 of 85 (62\%) prescreened bone marrow biopsies stained for BCMA by IHC met their prespecified threshold for eligibility, meaning more than a third of potentially eligible patients with MM would have been excluded (12). We did not require any specific level of BCMA as an eligibility requirement, and identified MM cell BCMA expression by flow cytometry in all subjects, consistent with recent data that flow cytometry is more sensitive than IHC for this purpose (28). Baseline BCMA intensity by flow cytometry did not correlate with either expansion or response in our study (Supplemental Figure 9 and Supplemental Figure 10), suggesting that excluding patients based on baseline BCMA expression is likely not necessary.
The observed dynamics of BCMA surface expression on MM cells, with the residual MM cells from several subjects in this study having significantly diminished BCMA intensity following CAR T cell therapy (Figure 5), highlights an important area for future research into resistance to CART-BCMA therapy. Down-modulation of BCMA expression was also observed in at least 1 subject in the NCI study (20), suggesting that it may be a common means of MM cell escape from BCMA-directed CAR T cell therapies. Surface BCMA expression subsequently increased in most subjects upon progression, suggesting a transcriptional or posttranslational mechanism, such as increased shedding from the cell surface. Alternatively, there may be immune selection for BCMA-dim/negative clonal variants, which are subsequently outcompeted by residual $\mathrm{BCMA}^{+}$clones upon loss of CART-BCMA cell activity. This suggests 
A

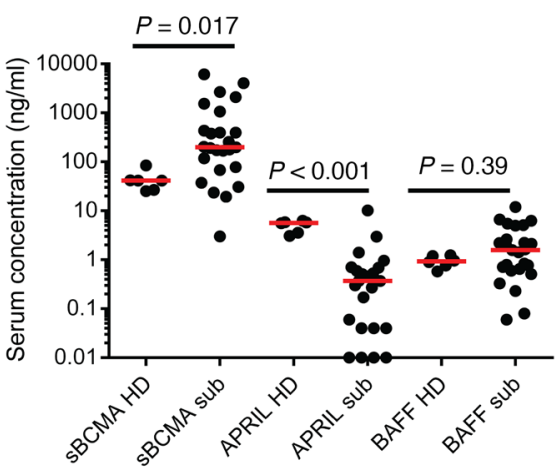

C
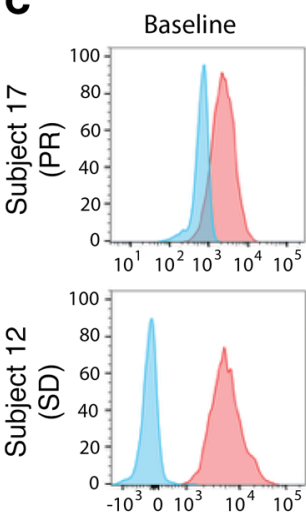

Day 28
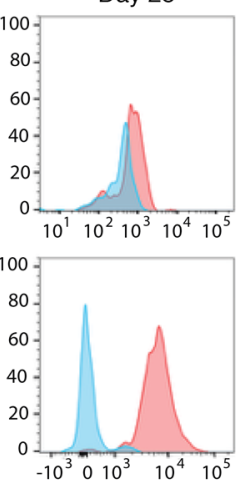

B

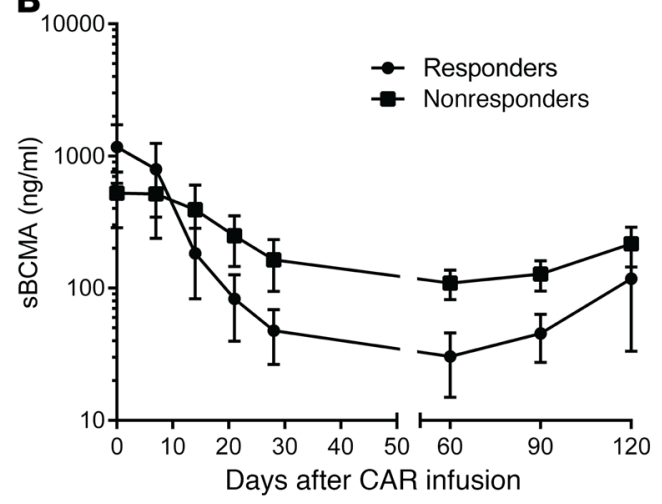

D
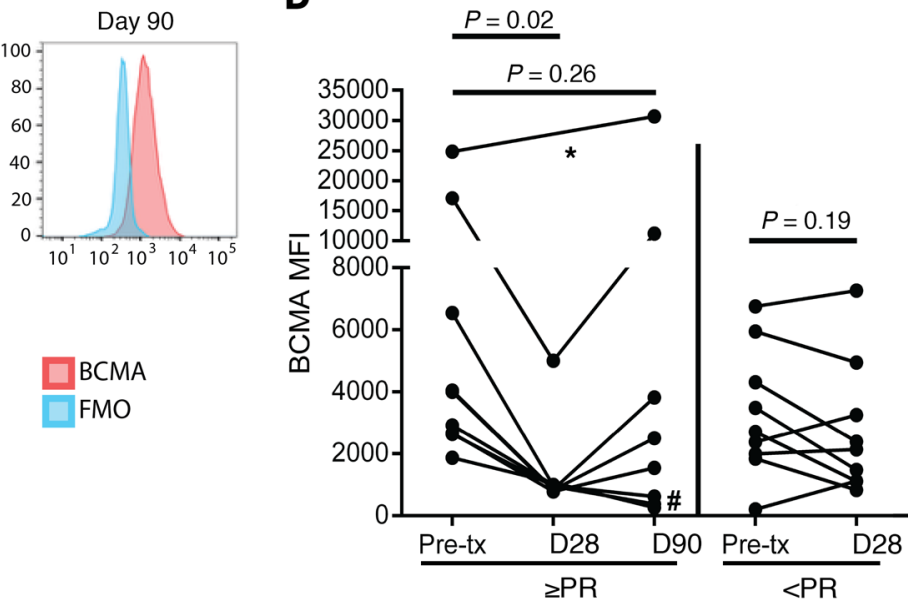

Figure 5. Soluble BCMA (sBCMA), BAFF, and APRIL concentration, and BCMA expression on MM cells before and after CART-BCMA infusions. (A) Baseline peripheral blood serum concentration of sBCMA and APRIL for subjects (sub) were significantly increased and decreased, respectively, compared with a panel of healthy donors (HD, $n=6)(P=0.017$, and $P<0.001$, respectively, Mann-Whitney). Baseline BAFF concentrations were not significantly different. Median concentrations are depicted by red lines. (B) Serial SBCMA concentrations decline after CART-BCMA infusions more significantly in hematologic responders (PR/VGPR/CR/sCR) than in nonresponders (MR/SD/PD) before day $28(P<0.001)$. After day 28 the slopes of the curves are not significantly different between groups $(P=0.429)$. The estimation was based on a linear random intercept mixed effects model on log ${ }^{10}$-transform sBCMA that included 2 piecewise linear splines connected at day 28; $P$ values were determined based on $z$ test for the regression coefficient of interest or a linear combination of the coefficients. Mean concentration ( $\mathrm{ng} / \mathrm{ml})+\mathrm{SEM}$ are depicted. (C) Representative examples of BCMA expression on MM cells by flow cytometry. See Supplemental Figure 9 for gating strategy. FMO, fluorescence minus one. (D) BCMA mean fluorescence intensity (MFI) on MM cells over time in 18 subjects with evaluable serial bone marrow aspirates. Median MFI was significantly different between pretreatment (pre-tx) and day 28 (D28) for responders (4000 vs. $944, P=0.02$, paired $t$ test) but not for nonresponders (2704 vs. $2140, P=0.19$ ). Median MFI was not significantly different between pre-tx and day 90 (D90) for responders (4000 vs. 2022, $P=0.26$ ). ${ }^{*}$ Subject 15 had no detectable MM cells at D28. \#Subject 03 had no detectable MM cells at D45 (D28 not done) and too few MM cells to characterize at D90. D164 marrow is depicted at D90 time point.

that most patients progressing after CART-BCMA will remain candidates for additional BCMA-targeted therapies.

Our prior studies of CD19-specific CAR T cells in CLL and ALL suggest that durability of remission is associated with long-term persistence of CAR T cells $(14,16)$. In this study, however, only 1 (subject 01) of the 3 long-term responders (subjects $01,19,33$ ) had detectable CART-BCMA cells by flow cytometry after day 60 (Supplemental Figure 4, Supplemental Figure 6). All 3 had detectable CAR T cells by qPCR at 3 months, but the amount of detectable BCMA CAR transgene (measured as copies/ $\mu$ g genomic DNA) in subjects 19 and 33 was similar to or less than several other patients who ultimately progressed between months 3 and 6 (Supplemental Table 6). Thus, in myeloma, CAR T cell persistence alone may not be enough to predict long-term durability of remission, and other factors, such as T cell functionality, antigen expression, and/ or immunosuppressive elements in the tumor microenvironment may play a role.

The primary toxicities of CAR T cells remain CRS and neurotoxicity. The frequency and severity of CRS in this study was similar to that reported in our CD19-targeted CAR T cell trials $(14,16)$, and was abrogated with IL-6 receptor blockade therapy. Though patient numbers are small, peak serum cytokine increases did not appear to differ significantly with or without Cy lymphodepletion, when CAR T cell dose was kept the same (cohort 1 vs. 3, Supplemental Figure 7). Interestingly, however, median peak fold-increases of IL-6 and several other cytokines (e.g., IFN- $\gamma$, IL-10, GM-CSF, IL-17) in this study were 1 to 2 orders of magnitude lower than that reported in the NCI BCMA CAR T cell study 

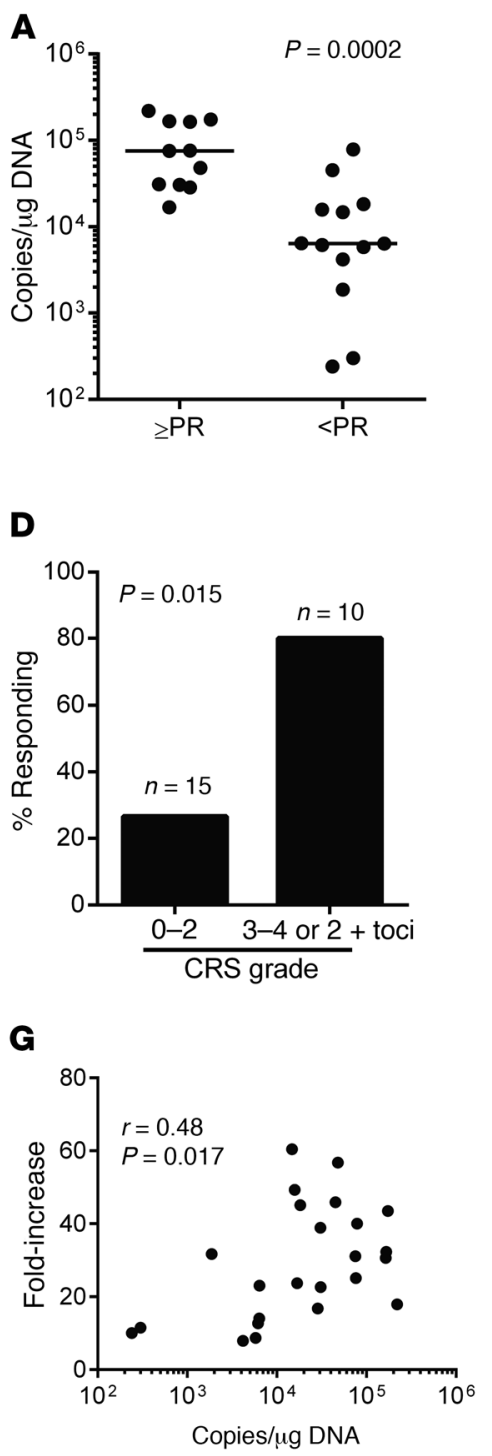
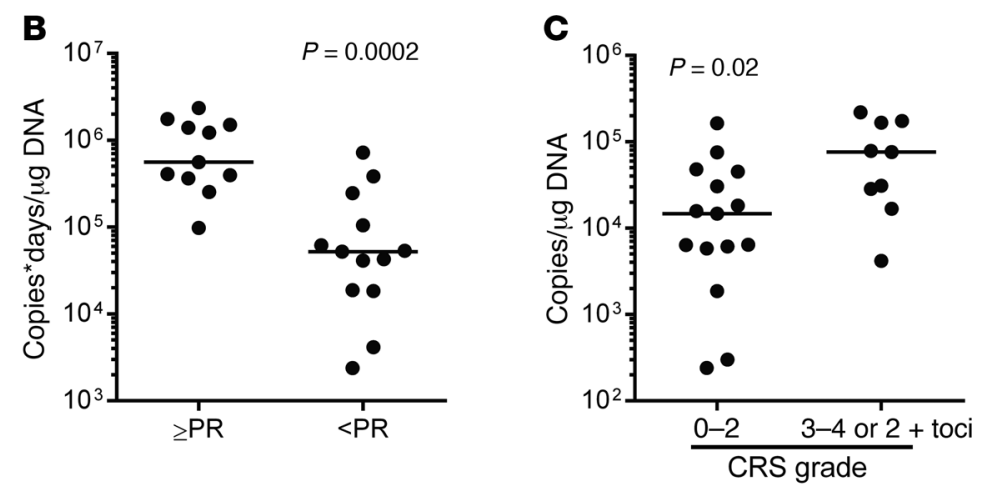

E
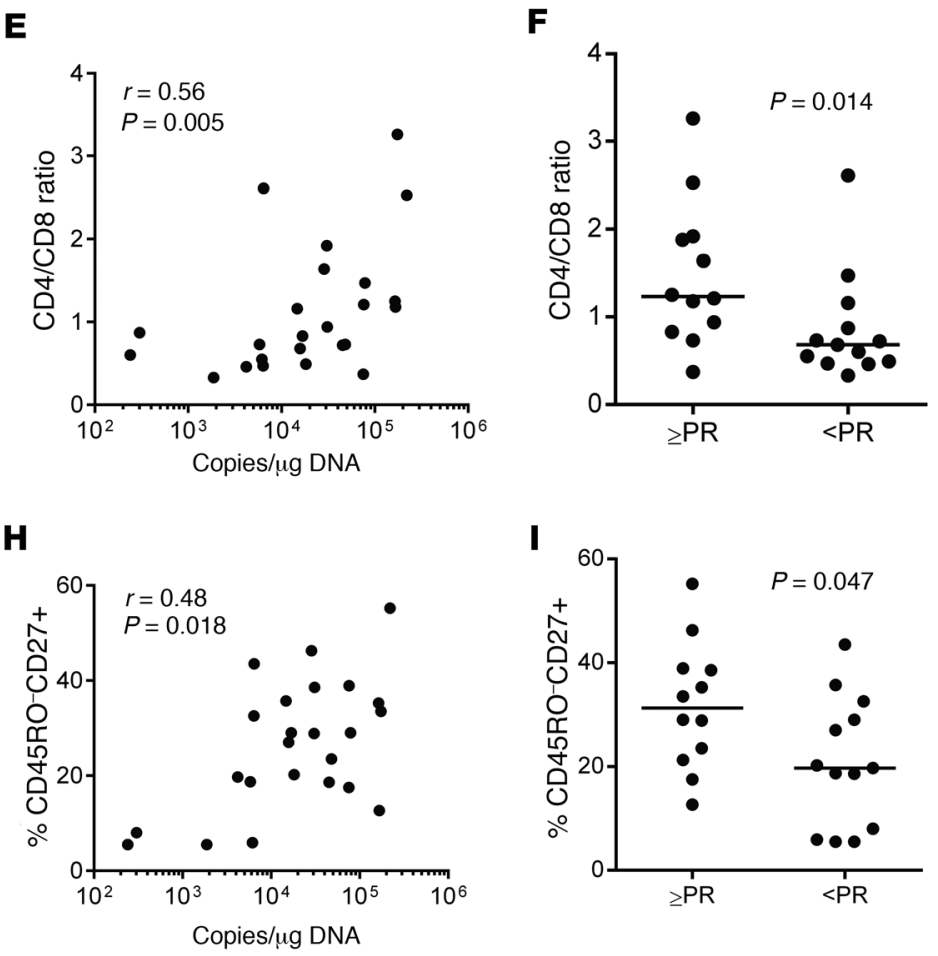

1

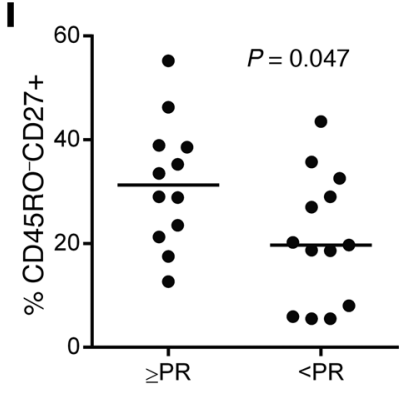

Figure 6. Predictors of in vivo CART-BCMA expansion and response. (A) Peak blood CART-BCMA expansion, as measured by qPCR, as well as (B) total CART-BCMA expansion over first 28 days (calculated as AUC), were both associated with clinical response. Greater peak CART-BCMA expansion (C) and response (D) were also associated with more severe CRS, defined as grade $3-4$ or grade 2 requiring tocilizumab. A higher ratio of CD4 ${ }^{+}$to $C D 8^{+} T$ cells (CD4/CD8 ratio) within the leukopheresis product, as determined by flow cytometry, also correlated with both peak expansion $(\mathbf{E})$ and response $(\mathbf{F})$, while in vitro proliferation, measured as fold-increase of seeded cells during manufacturing, correlated only with peak expansion (G), but not response $(P=0.54$, Mann-Whitney test, data not shown). (H-I) A higher proportion of $\mathrm{CD}^{+} \mathrm{T}$ cells within the leukapheresis product with a $\mathrm{CD}^{2} \mathrm{R}_{\mathrm{RO}}{ }^{-} \mathrm{CD} 27^{+}$phenotype was significantly associated with peak CART-BCMA expansion (H) and to a lesser degree, response (I). For A, B, C, F, and I, analysis was performed using Mann-Whitney test; lines represent median values. For $\mathrm{D}$, analysis was by Fisher's exact test. For $\mathrm{E}, \mathrm{G}$, and $\mathrm{H}$, analysis was done using Spearman correlation.

(20), despite a higher tumor burden in our study and despite the fact that our analysis of peak IL-6 levels included values obtained after tocilizumab administration, which is known to transiently further increase IL-6 levels due to binding of IL-6 receptor (17). One explanation for this difference may be the costimulatory domains used within the 2 CAR constructs, since CD28 domains, such as the one used in the NCI CAR construct, have been associated with more rapid CAR $T$ cell proliferation and cytokine release than 4-1BB domains (29), as used in our CAR construct. However, the small number of patients and multiple differences between the studies with regard to inclusion criteria, dose, schedule, and lymphodepletion regimen preclude definitive conclusions.
Neurotoxicity has been reported in up to $50 \%$ of subjects in some CAR T cell trials $(13,30,31)$, can occur concurrently with or subsequent to CRS, often does not improve with tocilizumab, and is reversible in most, but not all, cases. Neurotoxicity has been associated with early onset of CRS and rapid elevation of inflammatory cytokines both within the serum and CNS, perhaps leading to increased CNS vascular permeability (32). Consistent with this, we identified peak serum increases of IL-6, IFN- $\gamma$, and MIP-1 $\alpha$ as most associated with neurotoxicity in this study (Figure 4). Interestingly, neurotoxicity was also associated with peak fold-increase in IL-1RA, an endogenous inhibitor of the proinflammatory effects of IL- $1 \alpha$ and IL-1 $\beta$, which have been implicated in CAR T cell- 
associated neurotoxicity $(33,34)$. This perhaps reflects induction of an (ultimately ineffective) feedback mechanism in patients with neurotoxicity, and suggests that augmenting IL-1 blockade with the recombinant IL-1RA anakinra may have therapeutic benefit, as demonstrated in preclinical models $(33,34)$. Our experience demonstrating rapid reversal of a PRES-like syndrome in subject 03 suggests that cyclophosphamide may also be an option in steroid-refractory cases.

In summary, autologous $\mathrm{T}$ cells expressing a fully human BCMA-specific CAR could expand and induce objective responses, both with and without lymphodepleting chemotherapy, in subjects with advanced, refractory $\mathrm{MM}$, and represent a promising new therapeutic approach. The toxicity profile appears similar to that seen with CD19-directed CAR T cells in B cell malignancies. Challenges include disease progression during manufacturing, potential for antigen escape due to changes in BCMA expression, and durability of responses. Subsequent studies exploring less heavily pretreated/refractory patient populations, dual-antigen-targeting CAR constructs, novel lymphodepletion regimens or manufacturing protocols, and off-the-shelf CART products may further optimize the safety and long-term efficacy of this approach.

\section{Methods}

Study design and participants. Eligible subjects had relapsed and/ or refractory $\mathrm{MM}$ after at least 3 prior regimens, or 2 prior regimens if dual-refractory to a PI and IMID. Other key eligibility criteria at screening included ECOG (Eastern Cooperative Oncology Group) performance status of 0-2; serum creatinine no greater than $2.5 \mathrm{mg} /$ $\mathrm{dl}$ or estimated creatinine clearance at least $30 \mathrm{ml} / \mathrm{min}$; absolute neutrophil count at least $1000 / \mu \mathrm{l}$ and platelet count at least 50,000/ $\mu \mathrm{l}$ ( $\geq 30,000 / \mu \mathrm{l}$ if bone marrow plasma cells were $\geq 50 \%$ of cellularity); SGOT no more than 3 times the upper limit of normal and total bilirubin no greater than $2.0 \mathrm{mg} / \mathrm{dl}$; left ventricular ejection fraction at least $45 \%$; lack of active autoimmune disease; and lack of central nervous system involvement with myeloma. No prespecified level of BCMA expression on MM cells was required.

This clinical trial (NCTO2546167) was a phase I, single-center, open-label study. Initially, a standard $3+3$ dose-escalation design was used, exploring 3 sequential cohorts: cohort $1,1 \times 10^{8}$ to $5 \times 10^{8}$ CART-BCMA cells alone; cohort 2, Cy $1.5 \mathrm{~g} / \mathrm{m}^{2}$ plus $1 \times 10^{7}$ to $5 \times 10^{7}$ CART-BCMA cells; and cohort 3 , Cy $1.5 \mathrm{~g} / \mathrm{m}^{2}$ plus $1 \times 10^{8}$ to $5 \times 10^{8}$ CART-BCMA cells. The protocol was amended to expand each cohort to 9 treated subjects, to gain more information about the safety and efficacy of CART-BCMA cells both with and without lymphodepleting conditioning and at a higher $\left(1 \times 10^{8}\right.$ to $\left.5 \times 10^{8}\right)$ and lower $\left(1 \times 10^{7}\right.$ to 5 $\times 10^{7}$ ) dose. A subsequent amendment stopped enrollment in cohort 2 after 5 subjects due to suboptimal efficacy, and allowed up to 13 subjects in cohort 3; however, funding limitations ultimately ended enrollment after 11 treated cohort 3 subjects (total $n=25$ treated).

The study was designed by the lead authors and was conducted according to principles of Good Clinical Practice. An independent data safety monitoring board reviewed adverse event data and provided oversight. Data cut-off for analysis was July 9, 2018.

Procedures. After a 2-week washout from therapy (4 weeks for monoclonal antibodies), subjects underwent steady-state leukapheresis to collect T cells for CART-BCMA manufacturing. Antimyeloma therapy could resume during manufacturing until 2 weeks prior to first
CART-BCMA infusion. CART-BCMA cells were administered intravenously over 3 days (10\% of dose on day $0,30 \%$ on day 1 , and $60 \%$ on day 2), as described (16). The $30 \%$ or $60 \%$ dose could be held if subjects developed signs of CRS. Cy was administered 3 days prior to first CART-BCMA infusion. Clinical and laboratory assessments were performed as shown in Figure 1.

CART-BCMA cells were manufactured in the Clinical Cell and Vaccine Production Facility at the University of Pennsylvania, as described $(16,35)$. Briefly, autologous T cells were collected by leukapheresis and were stimulated with paramagnetic polystyrene beads coated with anti-CD3 and anti-CD28 monoclonal antibodies (36). Cells were transduced with a lentiviral vector encoding anti-BCMA scFv linked to $4-1 \mathrm{BB}$ and $\mathrm{CD} 3-\zeta$ signaling domains (15), as described (37), and were expanded ex vivo for 10 to 12 days in the presence of recombinant human IL-2. Release criteria have been previously described (35). The frequency of CD3, CD4, and CD8 cells was determined by flow cytometry in the seed culture at the beginning and end of manufacturing. Expansion and population doubling of seeded cells was measured by cell counting. After manufacturing, CART-BCMA cells were cryopreserved until time of infusion.

Data on adverse events (AEs) were collected from time of first CART-BCMA infusion (or Cy administration for cohorts 2 and 3). Toxicity grade was determined according to National Cancer Institute's Common Terminology Criteria for Adverse Events version 4.0, with the exception of cytokine release syndrome, which was graded as per the University of Pennsylvania CRS Grading System (Supplemental Table 1) (16). Myeloma responses were assessed by updated International Myeloma Working Group criteria (38).

Research sample processing, freezing, and laboratory analyses were performed in the Translational and Correlative Studies Laboratory at the University of Pennsylvania, as described $(14,16,35)$. CART-BCMA cells were quantified from peripheral blood or bone marrow samples by flow cytometry and quantitative PCR. Reagents and protocols for flow cytometry are described in the Supplemental Methods. Genomic DNA was isolated directly from whole blood or marrow aspirate, and qPCR analysis was performed using ABI TaqMan technology and a validated assay to detect the integrated CAR transgene sequence as per Supplemental Methods and described (35).

Serum cytokine levels were assessed on batched cryopreserved samples using the human cytokine magnetic 30-plex panel (LHC6003M) from Life Technologies, as described (14). Measurement of soluble BCMA, BAFF, and APRIL concentrations in serum was performed by ELISA using antibody sets for human BCMA (DY193), APRIL (DY884B), and BAFF (DT124-05) from R\&D Systems. See Supplemental Methods for details.

Flow cytometric assessment of MM cells, including BCMA expression, on fresh bone marrow aspirate material was adapted from the EuroFlow protocol (39) as described in Supplemental Methods. Flow cytometric assessment of T cell phenotype in cryopreserved leukapheresis specimens was performed and analyzed as described (18).

Outcomes. The primary objective was to evaluate the safety of CART-BCMA in patients with relapsed/refractory myeloma. The primary endpoint was incidence of study-related grade 3 or higher AEs, including DLTs. A DLT was defined as a serious and unexpected AE whose relationship to study therapy could not be ruled out, occurring within 4 weeks of receiving protocol therapy. Hematologic toxicity 
was not considered a DLT due to the refractory nature of the underlying disease and expected myelosuppression from cyclophosphamide. In addition, any death related to protocol treatment, as well as any expected grade 4 organ toxicity or grade 4 neurologic toxicity that did not resolve or improve to grade 2 or less within 4 weeks of onset, despite medical management, was also considered a DLT. Secondary objectives were to assess feasibility of manufacturing CART-BCMA cells, and clinical activity. Secondary endpoints were frequency of successful manufacturing, and clinical outcomes, including response rates, progression-free survival, and overall survival. Exploratory endpoints included CART-BCMA expansion and persistence in vivo; changes in concentration of serum cytokines and soluble BCMA, and expression of BCMA on MM cells.

Statistics. Statistical analysis of the study is primarily descriptive due to the pilot nature of the trial and small sample size of each cohort. Kaplan-Meier method was used to estimate duration of response, progression-free and overall survival, and the associated median survival times. Association between a binary endpoint (e.g., response) and a continuous factor (e.g., CAR T cell numbers) was evaluated using the Mann-Whitney test, or Kruskal-Wallis test if more than 2 groups were compared simultaneously. Association between a binary endpoint and a categorical factor (e.g., presence or absence of deletion 17p) was evaluated using Fisher's exact test. Spearman correlations were used to measure the correlations between 2 continuous variables. Significance of the Spearman correlation against the null hypothesis of no correlation was computed based on permutation. Modeling of longitudinal trajectories over time (e.g., for soluble BCMA concentrations) was done using a linear mixed effects model with random intercepts on the $\log ^{10}$ transformed scale. Piecewise linear spline was used to capture the nonlinear patterns before and after day 28. Because the statistical analyses performed here are exploratory and hypothesis-generating in nature, no adjustment of the $P$ values was made for multiple comparisons. Exact 2-sided $P$ values are reported when applicable. A $P$ value less than 0.05 was considered significant. Analysis was performed using Graphpad Prism version 6.0.

Study approval. The study was reviewed and approved by the University of Pennsylvania Institutional Review Board. The regulatory sponsor was the University of Pennsylvania. All subjects provided written informed consent prior to participation.

\section{Author contributions}

ADC, ALG, EAS, GP, CHJ, RI, and MCM designed the clinical trial. ADC, ALG, EAS, DTV, BMW, EL, KD, and AN enrolled, evaluated, and/or clinically managed subjects on the trial. DLS, MMD, and BLL directed patient $\mathrm{T}$ cell collection, supervised laboratory manufacture of CART cells, and/or approved clinical testing batch records for release of the CART cells. ADC, ALG, SFL, JJM, RMY, FC, JLB, IPM, BLL, CHJ, MMD, WTH, and MCM designed, analyzed, and/or interpreted correlative and laboratory data. ADC, ALG, EAS, DTV, BMW, and WTH analyzed and interpreted the clinical data. All authors participated in the drafting and/or review of the manuscript.

\section{Acknowledgments}

The authors thank the patients and their family members for their participation in the study and members of the Data Safety Monitoring Board. We thank L. Lledo, A. Marshall, J. Anderson, M. Truran, N. Kerr, R. Ferthio, T. Carey, L. Dengel, J. Knots-Miller, C. Desir, L. Caffee, L. O'Keefe, D. Fenderson, S. Le, and D. Vaughn for clinical trial, data management, and monitoring support, and D. Torigian for radiology review. We acknowledge and appreciate the assistance of J. Finklestein, F. Nazimuddin, C. Bartoszek, and B. Menchel for sample processing, I. Kulikovskaya and M. Gupta for qPCR analyses, D. Ambrose, L. Tian, and H. Parakandi for flow cytometry analyses, N. Kengle for Luminex cytokine analyses, V. Gonzalez and Y. Tanner for data management and quality control, and A. Lamontagne, A. Brennan, A. Malykhin, and members of the Clinical Cell and Vaccine Production Facility for cell manufacturing and testing. We acknowledge the medical and nursing staff of the Apheresis Unit at the Hospital of the University of Pennsylvania for their care and management of patients undergoing leukapheresis. This work was supported by a sponsored research agreement between the University of Pennsylvania and Novartis, as well as NIH grant 1P01CA214278 (to ADC, ALG, EAS, JJM, RMY, CHJ, MCM). ALG is supported by NIH grant K12CA076931 and a Conquer Cancer Foundation of ASCO Career Development Award.

Address correspondence to: Adam D. Cohen, Abramson Cancer Center, University of Pennsylvania, 3400 Civic Center Boulevard, PCAM-12 South, Philadelphia, Pennsylvania 19104, USA. Phone: 215.615.5853; Email: adam.cohen@uphs.upenn.edu.
1. Rickert RC, Jellusova J, Miletic AV. Signaling by the tumor necrosis factor receptor superfamily in B-cell biology and disease. Immunol Rev. 2011;244(1):115-133.

2. Frigyesi I, et al. Robust isolation of malignant plasma cells in multiple myeloma. Blood. 2014;123(9):1336-1340.

3. Tai YT, et al. Novel anti-B-cell maturation antigen antibody-drug conjugate (GSK2857916) selectively induces killing of multiple myeloma. Blood. 2014;123(20):3128-3138.

4. Carpenter RO, et al. B-cell maturation antigen is a promising target for adoptive T-cell therapy of multiple myeloma. Clin Cancer Res. 2013;19(8):2048-2060.

5. Seckinger A, et al. Target expression, generation, preclinical activity, and pharmacokinetics of the BCMA-T cell bispecific antibody EM801 for multiple myeloma treatment. Cancer Cell. 2017;31(3):396-410.

6. Laurent SA, et al. $\gamma$-Secretase directly sheds the survival receptor BCMA from plasma cells. Nat Commun. 2015;6:7333.

7. Sanchez E, et al. Serum B-cell maturation antigen is elevated in multiple myeloma and correlates with disease status and survival. Br J Haematol. 2012;158(6):727-738.

8. Moreaux J, et al. BAFF and APRIL protect myeloma cells from apoptosis induced by interleukin 6 deprivation and dexamethasone. Blood. 2004;103(8):3148-3157.

9. Tai YT, et al. APRIL and BCMA promote human multiple myeloma growth and immunosuppression in the bone marrow microenvironment. Blood. 2016;127(25):3225-3236.

10. Garfall AL, et al. Chimeric antigen receptor
T cells against CD19 for multiple myeloma. N Engl JMed. 2015;373(11):1040-1047.

11. Garfall AL, et al. Anti-CD19 CAR T cells with high-dose melphalan and autologous stem cell transplantation for refractory multiple myeloma. JCI Insight. 2018;3(8):120505.

12. Ali SA, et al. T cells expressing an anti-B-cell maturation antigen chimeric antigen receptor cause remissions of multiple myeloma. Blood. 2016;128(13):1688-1700.

13. Turtle CJ, et al. Immunotherapy of non-Hodgkin's lymphoma with a defined ratio of CD8+ and CD4+ CD19-specific chimeric antigen receptor-modified T cells. Sci Transl Med. 2016;8(355):355ra116.

14. Maude SL, et al. Chimeric antigen receptor $\mathrm{T}$ cells for sustained remissions in leukemia. N Engl JMed. 2014;371(16):1507-1517.

15. Bu DX, et al. Pre-clinical validation of B cell 
maturation antigen (BCMA) as a target for $\mathrm{T}$ cell immunotherapy of multiple myeloma. Oncotarget. 2018;9(40):25764-25780.

16. Porter DL, et al. Chimeric antigen receptor $\mathrm{T}$ cells persist and induce sustained remissions in relapsed refractory chronic lymphocytic leukemia. Sci Transl Med. 2015;7(303):303ra139.

17. Teachey DT, et al. Identification of predictive biomarkers for cytokine release syndrome after chimeric antigen receptor T-cell therapy for acute lymphoblastic leukemia. Cancer Discov. 2016;6(6):664-679.

18. Fraietta JA, et al. Determinants of response and resistance to $\mathrm{CD} 19$ chimeric antigen receptor (CAR) T cell therapy of chronic lymphocytic leukemia. Nat Med.2018;24(5):563-571.

19. Gattinoni L, Speiser DE, Lichterfeld M, Bonini C. $\mathrm{T}$ memory stem cells in health and disease. Nat Med. 2017;23(1):18-27.

20. Brudno JN, et al. T cells genetically modified to express an anti-B-cell maturation antigen chimeric antigen receptor cause remissions of poor-prognosis relapsed multiple myeloma. JClin Oncol. 2018;36(22):2267-2280.

21. Kay NE, et al. Blood levels of immune cells predict survival in myeloma patients: results of an Eastern Cooperative Oncology Group phase 3 trial for newly diagnosed multiple myeloma patients. Blood. 2001;98(1):23-28.

22. Dhodapkar MV, Krasovsky J, Osman K, Geller MD. Vigorous premalignancy-specific effector $\mathrm{T}$ cell response in the bone marrow of patients with monoclonal gammopathy. J Exp Med. 2003;198(11):1753-1757.

23. Suen $\mathrm{H}$, et al. Multiple myeloma causes clonal T-cell immunosenescence: identification of potential novel targets for promoting tumour immunity and implications for checkpoint blockade. Leukemia. 2016;30(8):1716-1724.

24. Dudley ME, et al. Adoptive cell therapy for patients with metastatic melanoma: evaluation of intensive myeloablative chemoradiation preparative regimens. JClin Oncol. 2008;26(32):5233-5239.

25. Noonan KA, et al. Adoptive transfer of activated marrow-infiltrating lymphocytes induces measurable antitumor immunity in the bone marrow in multiple myeloma. Sci Transl Med. 2015;7(288):288ra78.

26. Rapoport AP, et al. NY-ESO-1-specific TCRengineered $\mathrm{T}$ cells mediate sustained antigenspecific antitumor effects in myeloma. Nat Med. 2015;21(8):914-921.

27. Gattinoni L, Powell DJ, Rosenberg SA, Restifo NP. Adoptive immunotherapy for cancer: building on success. Nat Rev Immunol. 2006;6(5):383-393

28. Salem DA, et al. Quantification of B-cell maturation antigen, a target for novel chimeric antigen receptor T-cell therapy in myeloma. Leuk Res. 2018;71:106-111.

29. Milone MC, et al. Chimeric receptors containing CD137 signal transduction domains mediate enhanced survival of $\mathrm{T}$ cells and increased antileukemic efficacy in vivo. Mol Ther. 2009;17(8):1453-1464.

30. Turtle CJ, et al. CD19 CAR-T cells of defined CD4+:CD8+ composition in adult B cell ALL patients. JClin Invest. 2016;126(6):2123-2138.

31. Kochenderfer JN, et al. Chemotherapy-refractory diffuse large B-cell lymphoma and indolent B-cell malignancies can be effectively treated with autologous T cells expressing an antiCD19 chimeric antigen receptor. J Clin Oncol. 2015;33(6):540-549.

32. Gust J, et al. Endothelial activation and bloodbrain barrier disruption in neurotoxicity after adoptive immunotherapy with CD19 CAR-T cells. Cancer Discov. 2017;7(12):1404-1419.

33. Giavridis T, van der Stegen SJC, Eyquem J, Hamieh M, Piersigilli A, Sadelain M. CAR T cell-induced cytokine release syndrome is mediated by macrophages and abated by IL- 1 blockade. Nat Med. 2018;24(6):731-738.

34. Norelli M, et al. Monocyte-derived IL-1 and IL-6 are differentially required for cytokine-release syndrome and neurotoxicity due to CAR T cells. Nat Med. 2018;24(6):739-748.

35. Kalos $\mathrm{M}$, et al. $\mathrm{T}$ cells with chimeric antigen receptors have potent antitumor effects and can establish memory in patients with advanced leukemia. Sci Transl Med. 2011;3(95):95ra73.

36. Laport GG, et al. Adoptive transfer of costimulated $\mathrm{T}$ cells induces lymphocytosis in patients with relapsed/refractory non-Hodgkin lymphoma following CD34+-selected hematopoietic cell transplantation. Blood.2003;102(6):2004-2013.

37. Levine BL, et al. Gene transfer in humans using a conditionally replicating lentiviral vector. Proc Natl Acad Sci U S A. 2006;103(46):17372-17377.

38. Kumar S, et al. International Myeloma Working Group consensus criteria for response and minimal residual disease assessment in multiple myeloma. Lancet Oncol. 2016;17(8):e328-e346.

39. Flores-Montero J, et al. Next Generation Flow for highly sensitive and standardized detection of minimal residual disease in multiple myeloma. Leukemia. 2017;31(10):2094-2103. 